\title{
A knowledge model for the development of a framework for hypnogram construction
}

\author{
Ángel Fernández-Leal *, Mariano Cabrero-Canosa, Eduardo Mosqueira-Rey, Vicente Moret-Bonillo \\ Laboratory for Research and Development in Artificial Intelligence (LIDIA), Department of Computer Science, University of A Coruna, Spain
}

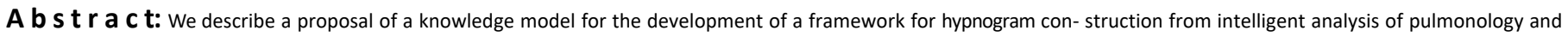

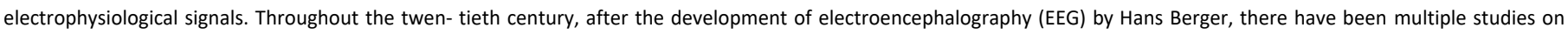

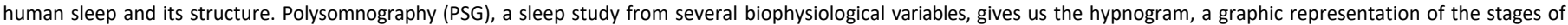

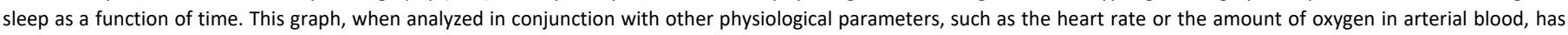

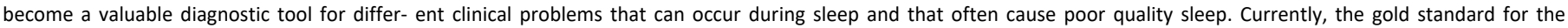

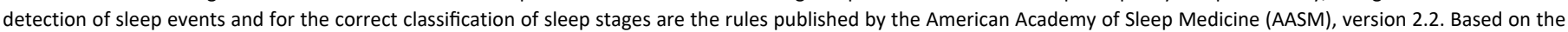

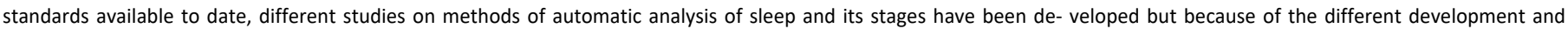

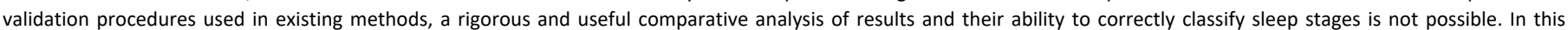

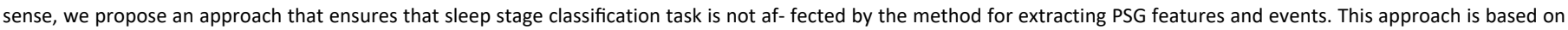

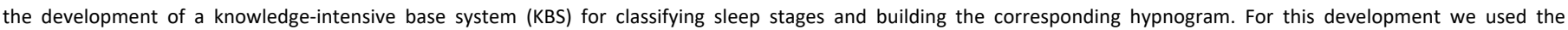

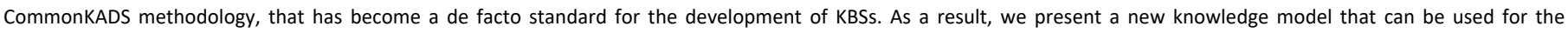

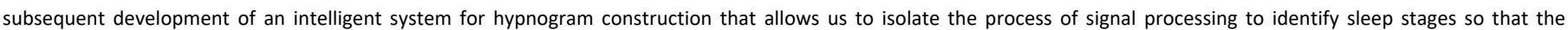
hypnograms obtained become comparable, independently of the signal analysis techniques.
\end{abstract}

Keywords: Artificial intelligence, Knowledge-based system, Hypnogram, CommonKADS

* Corresponding author. Facultade de Informática, Universidade da Coruña Campus de Elviña, s/n, 15071, A Coruña, Spain. Fax: + 34981167160.

E-mail addresses: angel.fleal@udc.es (Á. Fernández-Leal), mariano.cabrero@udce.s (M. Cabrero-Canosa), eduardo.mosqueira@udc.es (E. Mosqueira-Rey), vicente.moret@udc.es (V. Moret-Bonillo).

\section{Introduction}

Identification of the different phases through which the sleep of a person passes is useful in the diagnosis of certain sleep disorders, since some of these disorders occur during specific sleep stages. In the field of sleep disorders, polysomnography (PSG) is the main technique for analyzing a patient's biomedical signals such as brain and heart activity, eye and muscle movements and respiratory flow - plus another type of signal representing context information. Consequently, identifying sleep stages is a key task in the context of sleep studies conducted by PSG. This test has, how- ever, some drawbacks: it is expensive, uncomfortable for the patient and results are difficult to interpret.

To facilitate the analysis of sleep stages and their temporal sequencing, clinicians typically use a graphical representation of the chronology of different sleep stages, namely, the hypnogram. The hypnogram is built in the first quadrant of a Cartesian plane, where the X-axis represents time and the Y-axis represents sleep stages. The sequence of sleep stages is illustrated by a plot of horizontal and vertical lines, with the horizontal lines indicating the duration of a particular sleep stage and the vertical lines indicating changes in sleep stages. Fig. 1 shows an example of a typical hypnogram [1]. The hypnogram, when analyzed with other physiological PSG parameters - such as the heart rate or the amount of oxygen in arterial blood - has become a valuable clinical tool for physicians to diagnose a range of clinical problems that can occur during sleep and that sometimes result in poor quality sleep. Hypnogram con- 


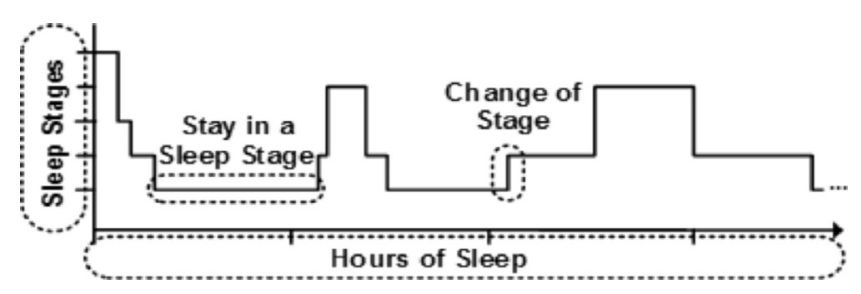

Fig. 1. Example of a hypnogram.

struction requires handling and analyzing large amounts of information and knowledge; hence, automating this task requires the building of a knowledge-intensive system (KBS).

When we build knowledge-intensive base systems, it is appropriate to use a comprehensive knowledge modeling methodology that should facilitate detailed analysis and comprehensive handling of knowledge-intensive tasks and processes; it should also provide for knowledge abstraction mechanisms that allows aspects related to implementation to be ignored. In this sense, we have proposed to build KBS for hypnogram construction using an appropriate methodology. Among the available methodologies for building KBSs, the CommonKADS methodology was selected for this work because it is a complete methodology that has become the de facto standard for the development of KBSs. Used extensively in a wide range of domains, it covers project management, organizational analysis and software and knowledge engineering aspects related to the development of the KBS, focusing, in particular, on modeling, reuse and risk management [2,3]. Its main advantage is that it provides a full model of an application, not just a model of the knowledge base.

Regarding clinical knowledge on sleep, the gold standard for the construction of hypnogram is the American Academy of Sleep Medicine (AASM) guidelines for the scoring of sleep and associated events [4] (version 2.2), including stages, arousals, movement and respiratory and cardiac events. In the construction of the proposed KBS, the terms specific to the domain and the sleep staging rules proposed by the AASM were used to obtain a correctly constructed hypnogram. The constructed hypnogram will improve the detection of events and diagnoses associated with sleep disorders and so will undoubtedly optimize timely therapy.

The rest of this paper is structured as follows: Section 2 provides a historical overview of the literature on sleep structure and hypnograms. Section 3 presents a summary of the most important studies of the automatic classification of sleep stages. Section 4 presents CommonKADS as the methodological framework used in this work. Sections 5, 6, 7 and 8 describe implementation of the concept level of our approach according to the CommonKADS methodology. Section 9 presents a short discussion. Finally, Section 10 presents a summary of conclusions.

\section{Historical background}

In 1929 the German neurologist Hans Berger demonstrated after developing electroencephalography (EEG) - that the electrical activity of the brain is different for sleeping and awake patients [5] and identified certain patterns associated with each of these two states, documenting and defining what are known as alpha waves and beta waves. With time, knowledge regarding the electrical activity of the brain grew and new patterns of electrical signals for sleep and wakefulness were identified, such as the delta waves described in 1936 by Walter [6] and the theta waves described in 1944 by Walter and Dovey [7].

In 1937 Loomis, Harvey and Hobart [8] determined that sleep is an active state with five distinct phases or stages labeled AE. Fig. 2 shows an example of the hypnogram of Loomis, who

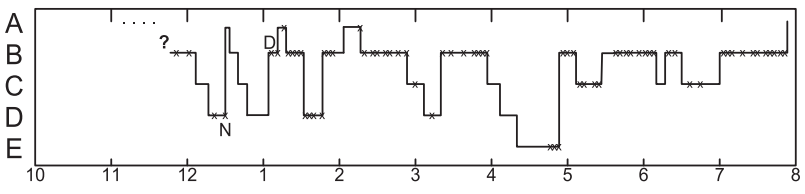

Fig. 2. An example of Loomis' hypnogram.

suggested that it was necessary to take into account 2 characteristic patterns of brain signals to correctly identify and classify sleep phases, namely, sleep spindles (also known as sigma waves) [9] and K complexes [10].

Independently of Loomis, Liberson [11] documented, in 1944, vertex sharp waves (VSW), a particular pattern of sleep that occurs early on in the night at the end of sleep onset and just before the onset of sleep spindles and $\mathrm{K}$ complexes.

Kleitman and Aserinsky [12] subsequently discovered rapid eye movements (REM) and Kleitman and Dement [13] discovered the recurring pattern of REM and non-REM (NREM) sleep, with NREM sleep divided into 4 stages numbered 1, 2, 3 and 4 . In stages 3 and 4 sleep becomes deeper. REM sleep appears to be associated with stages 5 and 1 .

This new classification of sleep stages introduced changes in the representation of hypnograms, giving them the fundamental form known to us today. Fig. 3 shows an example of the Dement and Kleitman hypnogram, with sleep stages represented on the vertical axis (awake (A) and the 4 stages) and time represented on the horizontal axis. The thick bars immediately above the EEG lines indicate periods during which REM is observed. Longer vertical lines indicate major movements (whole body position changes) and shorter lines represent minor movements. The arrows indicate the end of one EEG cycle and the beginning of the next.

Berger, Olley and Oswald [14] and Schwartz [15] subsequently improved identification of the REM stage, describing sawtooth waves and their temporary association with series of REM.

However, in the 1950s the lack of standards for classifying and identifying sleep phases made it difficult to compare results from sleep studies. In 1961, the International Federation of Electroencephalography and Clinical Neurophysiology Committee proposed unifying the terminology used in sleep studies [16] and in 1968, a committee headed by Rechtschaffen and Kales [17] established guidelines to standardize sleep stage classification - called the R\&K paradigm - that included parameters, techniques and wave patterns obtained from EEG, electro-oculography (EOG) and electromyography (EMG). The guidelines also indicated analyzing this set of signals in epochs of 20 or $30 \mathrm{~s}$. This approach, by specifying the fundamental characteristics of sleep stages, allowed for further standardization of hypnograms.

Nonetheless, there were difficulties in implementing the R\&K rules, especially in computing the classification of sleep, given certain ambiguities. Consequently, in 2001 the Sleep Computing Committee of the Japanese Society of Sleep Research (JSSR) [18] proposed additional criteria and corrections to the R\&K rules aimed at improving sleep stage classification and identification. In 2006 the Scoring of Polysomnographic Recordings Task Force of the German Sleep Society (DGSM) [19] conducted a study aimed at refining the identification of important signals in EEG patterns.

In 2007, the American Academy of Sleep Medicine (AASM) [20] also carried out a study in order to establish more precise definitions and more appropriate rules for the detection of sleep events and for the correct classification of sleep stages. Sleep stages were thus redefined as follows: (a) stage W (awake), (b) stage N1 (NREM 1), (c) stage N2 (NREM 2), (d) stage N3 (NREM 3 ), and (e) stage R (REM). Note that stage N3, representing slowwave sleep (SWS), replaces the previous nomenclature referring to stages 3 and 4 . 


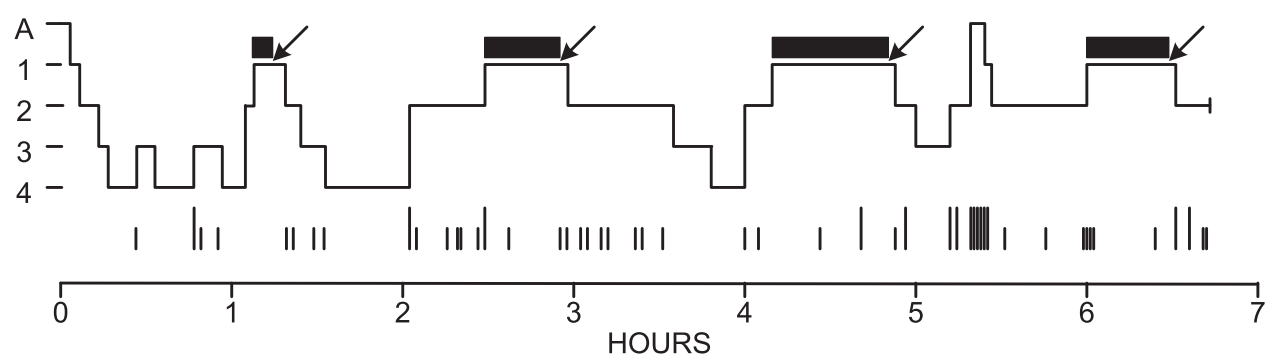

Fig. 3. Example of the Dement and Kleitman hypnogram.

Table 1

Sleep stage classification according to Loomis, Dement and Kleitman, Rechtschaffen and Kales (R\&K) and AASM-2015 and the corresponding EEG activity.

\begin{tabular}{llll}
\hline Loomis & Dement and Kleitman & R\&K & AASM-2015 \\
\hline & & Stage W & EEG activity \\
& & Movement time (MT) & Beta, Alpha \\
Stages A and B & Stage 1 & Stage 1 (S1) & Stage N1 \\
Stage C & Stage 2 & Stage 2 (S2) & Stage N2 \\
Stages D and E & Stage 3 & Stage 3 (S3) & Stage N3 The, Theta, Vertex sharp waves \\
& Stage 4 & Stage 4 (S4) & Theta, Delta \\
& Stage 1+REM (after the first stage 1) & Stage REM & Stage R \\
\hline
\end{tabular}

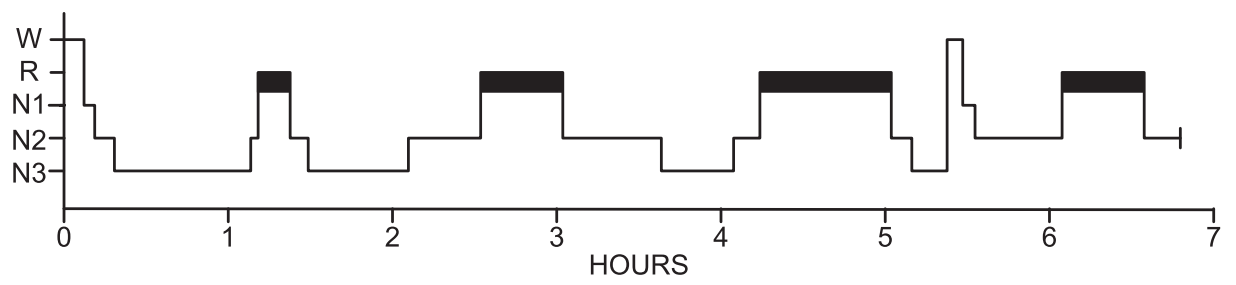

Fig. 4. Example of a hypnogram reflecting the AASM-2015 standard.

To identify and classify sleep stages, $30 \mathrm{~s}$ intervals are used in such a way that a stage is assigned to each interval. If two or more stages co-occur in the same interval, the interval is assigned to the stage of longer duration. Table 1 shows correspondence between the Loomis, Dement and Kleitman, R\&K and AASM sleep stage classifications and characteristic EEG activity at each stage [1].

In 2012, 2014 and 2015 the AASM published versions 2.0 [21], 2.1 [22] and 2.2 [4], respectively, of the 2007 criteria. The latest revision - which introduced stage $\mathrm{T}$ (transitional), analogous to the previously used 'indeterminate sleep' terminology in sleep staging for infants - is currently the gold standard for identifying and classifying sleep stages and for constructing hypnograms. Fig. 4 shows an example of a hypnogram built according to the AASM-2015 standard.

\section{Automatic sleep staging}

The advent of computers in the second half of the twentieth century and the rapid increase in their capabilities led to the realization of numerous studies on methods of automatic analysis of sleep and its stages. The two main aspects of the implemented approaches are [23]: (a) most of the work performed signal characteristic extraction by frequency analysis and frequency over time using discrete wavelet transform (DWT), Huang Hilbert transform (HHT), and fast Fourier transform (FFT) [24-27], and (b) different parametric and non-parametric methods have been applied in the classification of sleep events and stages, including random forest classifiers, artificial neural networks (ANN), fuzzy logic, nearest neighbor, linear discriminant analysis (LDA), support vector machines (SVM) and kernel logistic regression (KLR) [28-36].

Moreover, the results of a number of studies indicate that correct identification of characteristic signal patterns (especially sleep spindles [37], K complexes [38,39], VSW and sawtooth waves) and of events such as arousals (abrupt shifts in EEG frequency during sleep [40]) is critical for correct classification of sleep stages.

As a result of these circumstances, the different recording conditions and validation procedures used in existing methods mean that a rigorous and useful comparative analysis of results and of their ability to correctly classify sleep stages is not possible.

Consequently, we propose an approach that ensures that sleep stage classification is not affected by the method for extracting PSG features and events, based on the development of an intelligent module for classifying sleep stages and building the corresponding hypnogram. This module is integrated within a larger PSG system (either in a sleep unit or outpatient clinic) that detects and identifies characteristic signal patterns to be passed to the classifier module. As mentioned, we use the CommonKADS methodology to ensure adequate representation of the sleep macrostructure. The inclusion of this intelligent sleep classifier module in an integrated system results in a perfectly autonomous product potentially ready for commercialization.

\section{Methodological framework: CommonKADS}

CommonKADS is a complete methodology for the development of KBSs that covers project management, organizational analysis and software and knowledge engineering aspects related to the development of the KBS, focusing, in particular, on modeling, reuse and risk management.

CommonKADS can be viewed as a structured grouping of knowledge that reflects all the necessary aspects for the KBS to succeed within the organizational context in which the system will be deployed. To reflect these aspects, CommonKADS offers six models: organization, tasks, agents, knowledge, communication and design. All the models are interrelated and can be constructed using special CommonKADS forms provided for their preparation. 


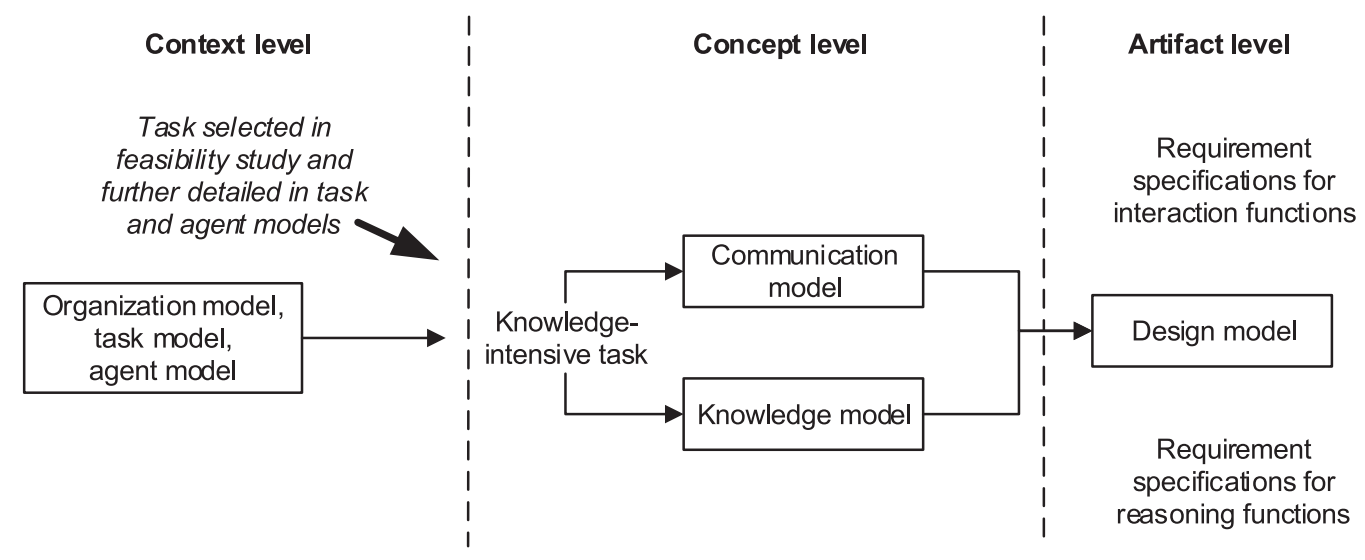

Fig. 5. Concept level model relationship with other models in the CommonKADS methodology.

The models reflect three levels specifying three different system aspects, as illustrated in Fig. 5:

(1) The context level responds to the question 'why develop a KBS' and includes the organization, task and agent models. The institution in which the project is developed is studied and described (in our case, either a sleep unit or an outpatient clinic). Also analyzed is why a KBS is being developed, its costs and benefits and its impact.

(2) The concept level responds to the question 'what is the nature and the structure of knowledge and communication involved in the task'. What the system should do is determined, without indicating how it should do this. The complete conceptual modeling process includes the development of the knowledge and communication models. In CommonKADS the knowledge model specifies - without referring to the implementation details - the structure of the information and the knowledge required for the application. The communication model specifies requirements related to interaction between the KBS and the other software components. The input is knowledge previously identified in the viability study implemented by the organization model and the output is the input to the design model.

(3) The artifact level responds to the question how should knowledge be implemented in a computer system and what is the architecture of the KBS'. At this level, technical aspects of implementation, comprising the design model, are central.

We describe the concept level, since at this level the structure of the information and knowledge required by the KBS is specified without reference to implementation details, meaning that the design can be implemented in any development environment.

Thus, once the context for the hypnogram is defined, the conceptual framework for completing hypnogram construction is described. The process of constructing knowledge models includes three main stages: identification, specification and refinement.

\section{Knowledge model: identification stage}

The goal is to examine and collect available knowledge components and adapt them so that they can be reused later. This process includes two activities: the search for sources of information and the listing of possible components of knowledge to be reused.

The knowledge-intensive task (and its related information) is implemented after the context model. In our case, the task that will focus the development of the knowledge model is 'hypnogram construction' .

After analyzing the information associated with the hypnogram construction task, we identified the next terms specific to the domain, as follows:
- Sleep: a physiological state associated with inaction and suspension of the senses and of all voluntary movement.

- Hypnogram: a graphical representation of the chronology of different sleep stages.

- Sleep stages:

- Stage W (Wakefulness)

- Stage NREM

- Stage N1 (NREM 1)

- Stage N2 (NREM 2)

- Stage N3 (NREM 3)

- Stage R (REM)

- Stage T (Transitional - only in infants)

- General sleep stage scoring strategy:

- Score sleep stages in $30 \mathrm{~s}$ sequential epochs commencing at the start of the study.

- Assign a stage to each epoch.

- If two or more stages co-occur during a single epoch, assign the stage reflecting most of the epoch.

- Score in accordance with the following definitions for EEG frequencies: - Slow wave activity: frequency of $0.5-2.0 \mathrm{~Hz}$ and minimum amplitude of $75 \mu \mathrm{V}$ peak to peak in frontal derivations

- Delta waves are $0-3.99 \mathrm{~Hz}$

- Theta waves are $4-7.99 \mathrm{~Hz}$

- Alpha waves are $8-13 \mathrm{~Hz}$

- Beta waves are greater than $13 \mathrm{~Hz}$

- Sleep onset: The start of the first epoch scored as any stage other than stage W. In most subjects this will usually be the first epoch of stage N1.

- Scoring arousals: Score arousal during sleep stages NI, N2, N3, or R if there is an abrupt shift of EEG frequency including alpha, theta and/or frequencies greater than $16 \mathrm{~Hz}$ (but not spindles) that lasts at least $3 \mathrm{~s}$, with at least $10 \mathrm{~s}$ of stable sleep preceding the change. Scoring of arousal during REM requires a concurrent increase in submental EMG lasting at least $1 \mathrm{~s}$.

- Taxonomy of characteristic signal patterns. Fig. 6 shows a classification of the main signal patterns used in the construction of hypnograms.

\section{Knowledge model: knowledge specification stage}

This stage is developed in three phases. First a suitable task template is selected. Second, an initial specification of the schema of the knowledge domain is carried out (independently of the problem to be solved). Finally, the knowledge model specification is completed.

\subsection{Task template selection}

This refers to an attempt to reuse one of the templates described in the CommonKADS libraries. There are two main types of tasks (and templates): analytic and synthetic, and for the characteristics of the task at hand synthetic is more adequate, and among the several templates of this type, the configuration template is chosen, adapting it to our restrictions.

The purpose of the configuration template is to find a combination of components that meets the requirements and restrictions 


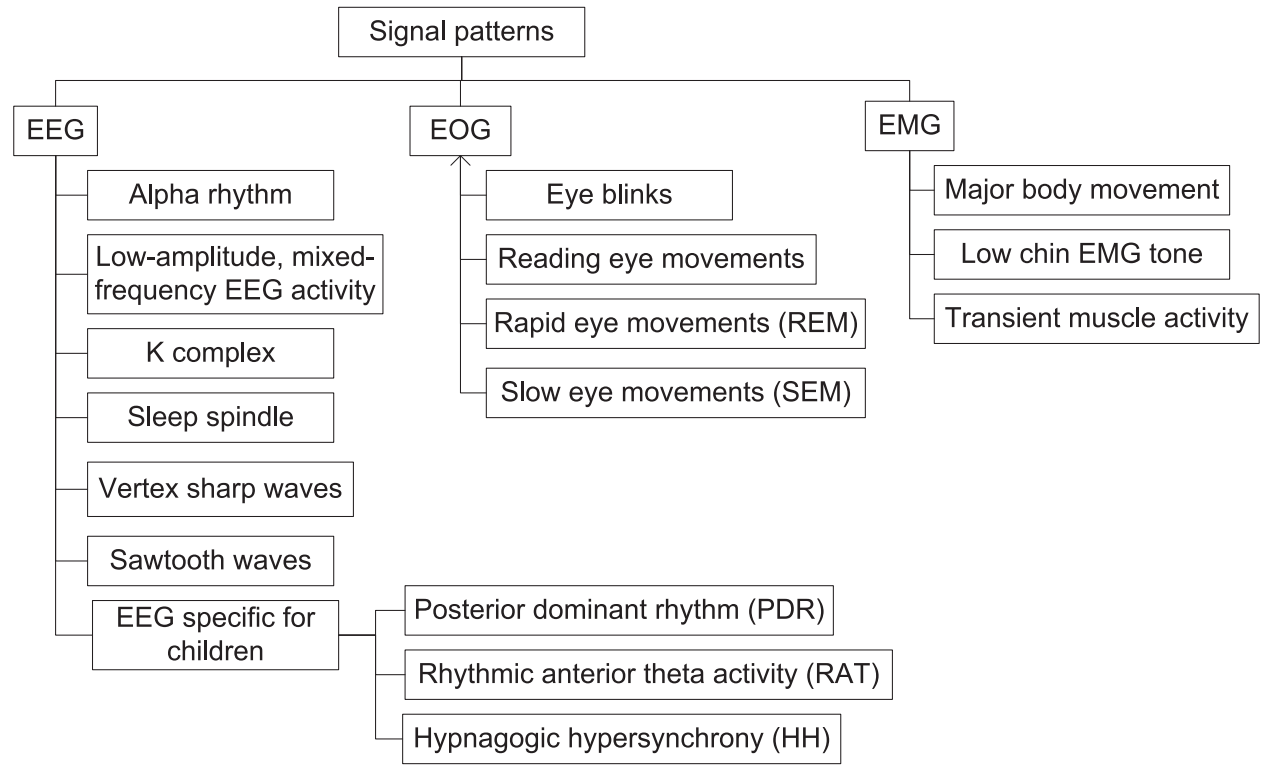

Fig. 6. Taxonomy of characteristic signal patterns.

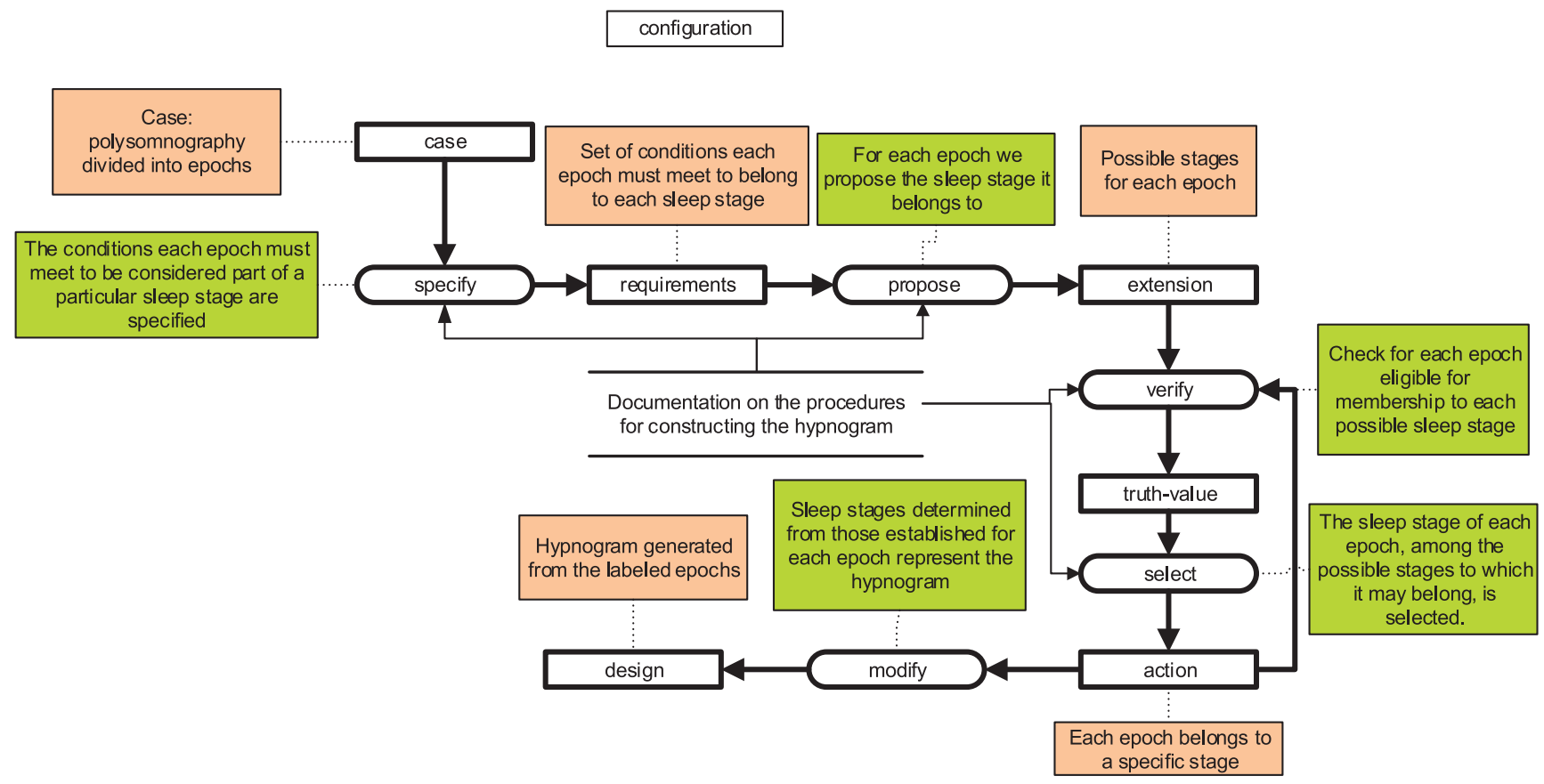

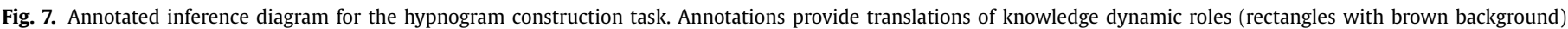

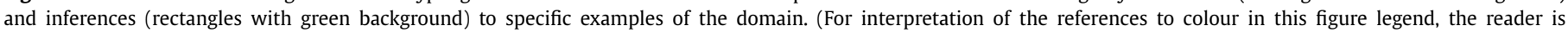
referred to the web version of this article.)

imposed. The following are selected from the set of inferences that make up this template:

- A specify inference that establishes the requirements for the substructures which compose each structure.

- A propose inference that identifies types of substructure in the corresponding major structure (extension).

- A verify inference that verifies that the requirements for each proposed substructure are met and, if so, chosen is the most appropriate substructures among those with positive values.

- A select inference that is usually a simple selection of one of the options generated by the previous function.

- A modify inference that adds the proposed structure to the case study.
After the study, an annotated template was developed to verify that template selection was correct. Thus, specific examples were assigned to the dynamic roles to check if the assignments were forced or difficult to understand. We concluded that the templates were suitable, a hypothesis that was further checked in the knowledge modeling phase.

In Fig. 7, the inferences applied to our specific domain are identified and described.

\subsection{Initial specifications}

This category represents the relevant knowledge of object modeling in the application domain and independently of the problem to be solved. 


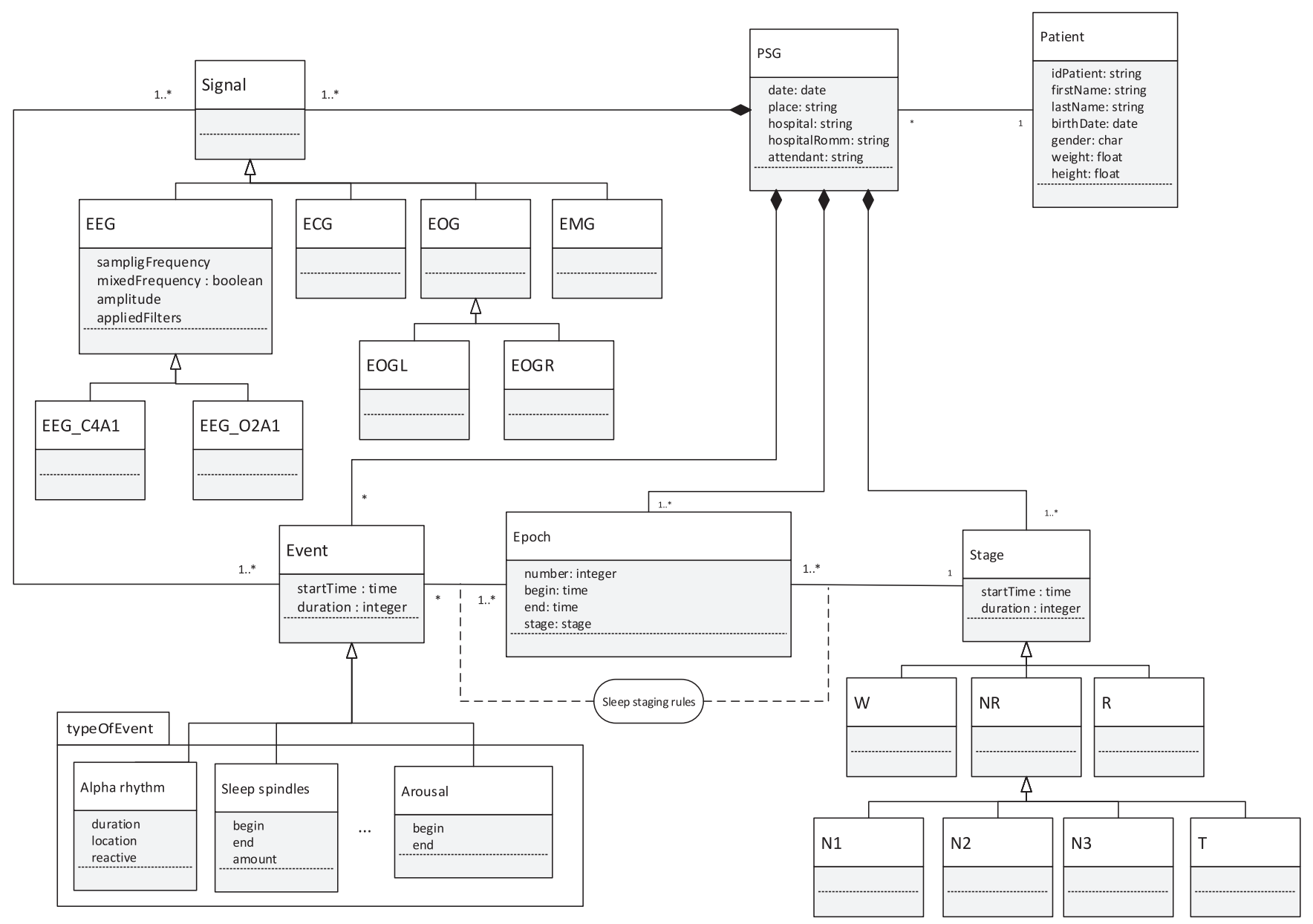

Fig. 8. UML diagram corresponding to the knowledge schema.

For the purposes of this specification, this category is divided into two parts: the domain scheme and the knowledge base. The first part includes the general terminology used to describe the knowledge of the particular domain, while the second extends the first part with specific facts of this domain.

CommonKADS provides three basic constructors to specify the domain schemas: (a) concepts, (b) relationships and (c) types of rules.

\subsubsection{Concepts}

Concepts (or classes of concepts) are used to define collections of objects which have similar characteristics. The definition of concept bears some similarity to the object classes referred to in approaches such as the object-oriented approach. However, the main difference is that, in this context, no functional information (methods or operations) is included in the descriptions of the different items.

One of the salient features of the knowledge model in CommonKADS is the ability to specify generalization and specialization relationships between concepts. This specialization/generalization relationship allows us to represent a taxonomy that groups domain concepts. Moreover, all these concepts have to be consistent with the terms used in the domain.

We identified the following concepts. A patient will undergo several PSGs to diagnose sleep-related problems. Each PSG is a composite of EEG, ECG, EOG and EMG signals and generally refers to eight hours of sleep. The PSG is divided into $30 \mathrm{~s}$ intervals called epochs and can thus be viewed as being composed of epochs. An analysis of PSG signals enables representative events for diagnosis to be identified (for example, K-complexes, sleep spindles, etc.), so the PSG can also be viewed as being composed of events. Additionally, since an event may last more than $30 \mathrm{~s}$, it may appear in more than one epoch.

The analysis of the different events that occur in the epochs determines the sleep stage to which each epoch belongs and thus establishes sleep stages and their sequence. So it follows that a PSG is also a composite of sleep stages.

\subsubsection{Relationships}

Once the concepts have been identified we describe the relationships that exists between them.

We have seen that a PSG is a composite of signals, events, epochs and sleep stages. One or more signals can be used to detect one or more relevant events for the construction of the hypnogram.

Several events may occur in a single epoch and an event may occur over one or more epochs.

One or more epochs may belong to a particular stage of sleep, and there may be multiple occurrences of the same type of stage during sleep.

\subsubsection{Types of rules}

In order to define the different types of rules in a domain schema, we need to represent a precedent and a consequent, in which we indicate the concepts for whose instances logical expressions must be defined. In our case we considered sleep staging rules (as per AASM-2105) which determine the sleep stage to which each epoch belongs to depending on the events in each epoch. 
Fig. 8 depicts the UML diagram of the framework proposed for hypnogram construction.

\subsection{Complete knowledge model specification}

To complete the model, a 'middle-out' approach is used [3] because the system adapts well to the inference diagram described above. This is because we start with the inference diagram for the selected template and then complete the task knowledge, domain knowledge and inferential knowledge that makes the translation between the inference and domain roles.

\subsubsection{Task knowledge}

As discussed in the previous paragraph, due to the complexity of the task of "construction of the hypnogram", it was searched for a template appropriate for it. The hierarchical breakdown of the subtask can be seen in Fig. 9.

The description using the CommonKDAS conceptual modeling language (CML) of the task of the hypnogram construction and its method is as follows:

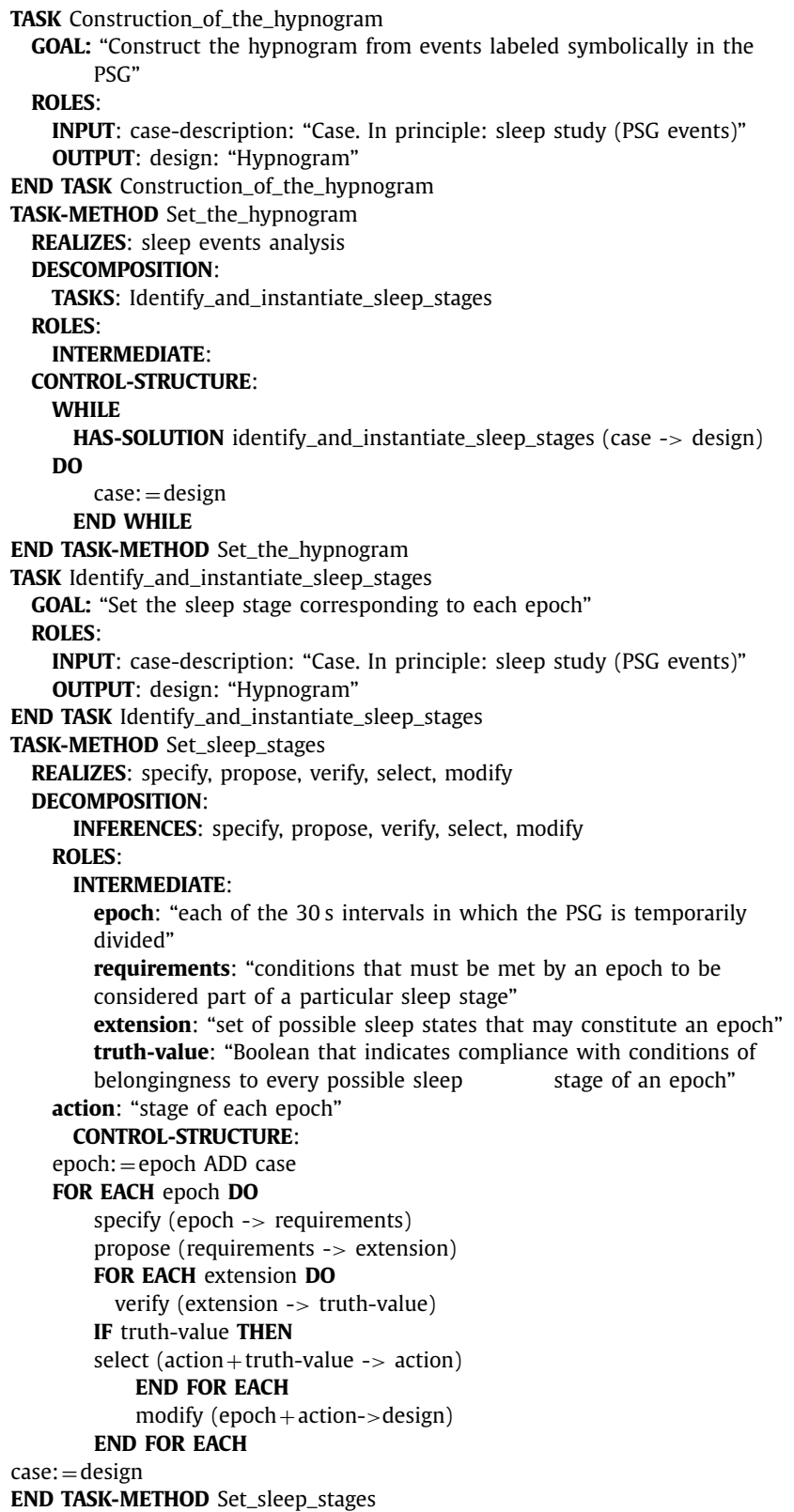

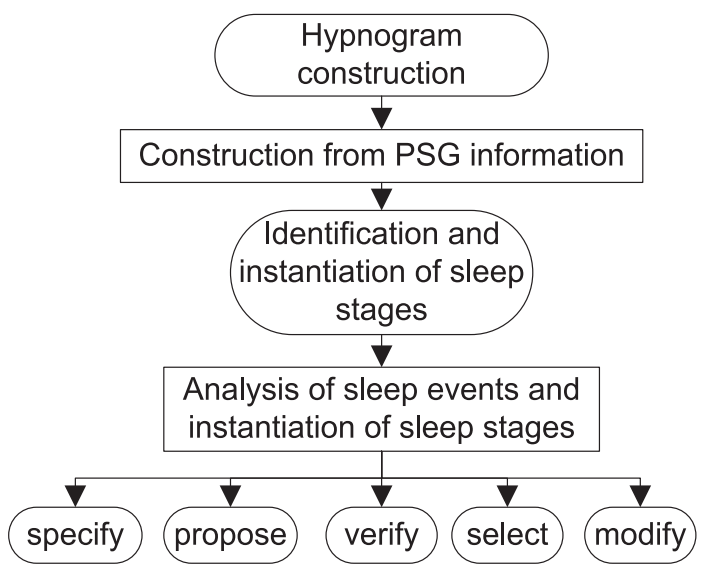

Fig. 9. Hierarchical breakdown of the hypnogram construction task (task structure).

\subsubsection{Domain knowledge: sleep staging rules}

The algorithm for scoring sleep stages is represented in Fig. 10a. For each epoch, the domain knowledge determines, if possible in view of available evidence, the most probable sleep stage. A second execution of the algorithm determines sleep stages for unclassified epochs between epochs classified definitively as sleep stages. In the reasoning process, definitive sleep stages act as inferential contexts. The final result is the hypnogram, that is, a graphical representation of all these sleep stages.

The selection process of the most probable sleep stage, taking into account the available evidence, follows the strategy represented in Fig. 10b. Even though different sleep stages initially are candidates for applying domain knowledge, a fuzzy reasoning process ultimately selects the definitive sleep stage.

Domain knowledge in the schema is essentially represented as four types of production rules that reflect the AASM-2015 scoring guidelines: (a) a general scoring rule; (b) a definitive or start-stage scoring rule; (c) a continuation stage scoring rule; and (d) a transition or end-stage scoring rule:

- General scoring rule: A rule that defines a general criterion for scoring epochs, depending on whether the patient is an adult, child or infant.

Example for an adult patient: Assign a stage to each epoch $(30 \mathrm{~s}$ section of PSG recording) from the study outset. If two or more stages co-occur in a single epoch, assign the stage reflecting most of the epoch.

- Definitive-stage scoring rule: A rule that clearly determines the stage of an epoch.

Example for an adult patient: Assign definitive stage $\mathrm{R}$ if there is low amplitude mixed frequency EEG activity without $\mathrm{K}$ complexes or sleep spindles AND low chin EMG tone for most of the epoch concurrent with REM AND REM at any position within the epoch. The CML specification is as follows:

VAR A,Q,R,S,T,U,V: facts

Q.name = "Low amplitude mixed frequency" AND

Q.duration $>0$ AND

R.name $=$ "K complex" AND

R.amount $=0$ AND

S.name $=$ "Sleep spindle" AND

S.amount $=0$ AND

T.name $=$ "Chin EMG muscle tone" AND

T.level = "low" AND

T.duration $>15$ s AND 


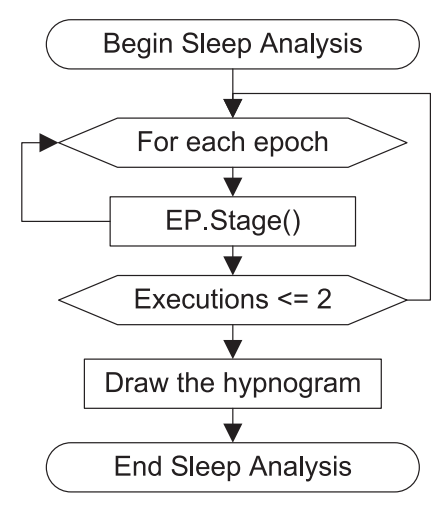

(a)

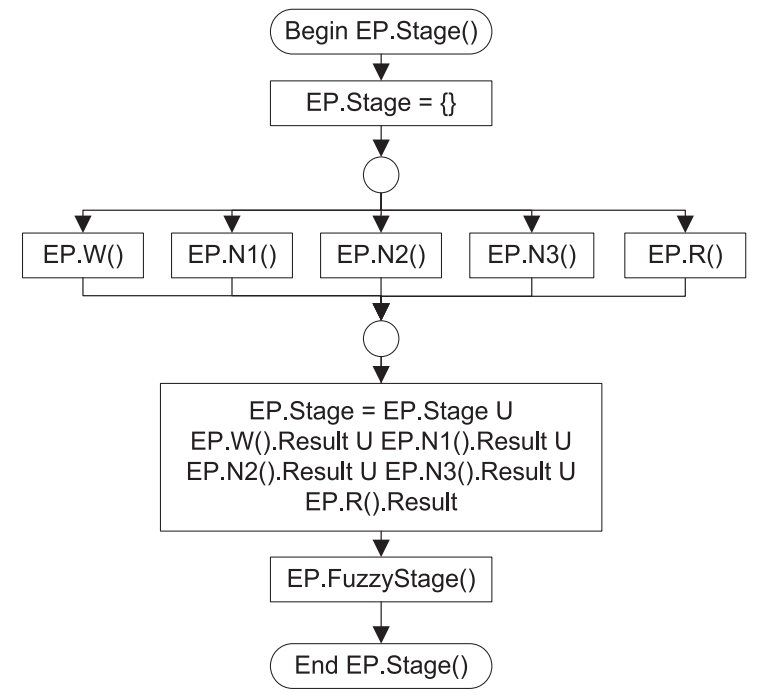

(b)

Fig. 10. a) Flow diagram of the general strategy for scoring sleep stages. b) Flow diagram of the fuzzy strategy for a given epoch (EP) determining its definitive sleep stage.

\author{
Uname = "Eye movements type" AND \\ U.REM $=$ True \\ IMPLIES \\ A.stage $=\mathrm{R}$
}

- Continuation-stage scoring rule: A rule that establishes criteria for scoring a stage contiguous to or preceding a definitive stage.

Example for an adult patient: In the absence of REM, score segments preceding or contiguous to an epoch of definitive stage $R$ as stage $\mathrm{R}$ if there is low amplitude mixed frequency EEG activity without $\mathrm{K}$ complexes or sleep spindles, there is low chin EMG tone, there is no intervening arousal and there is no SEM following an arousal or stage $\mathrm{W}$. The CML specification is as follows:

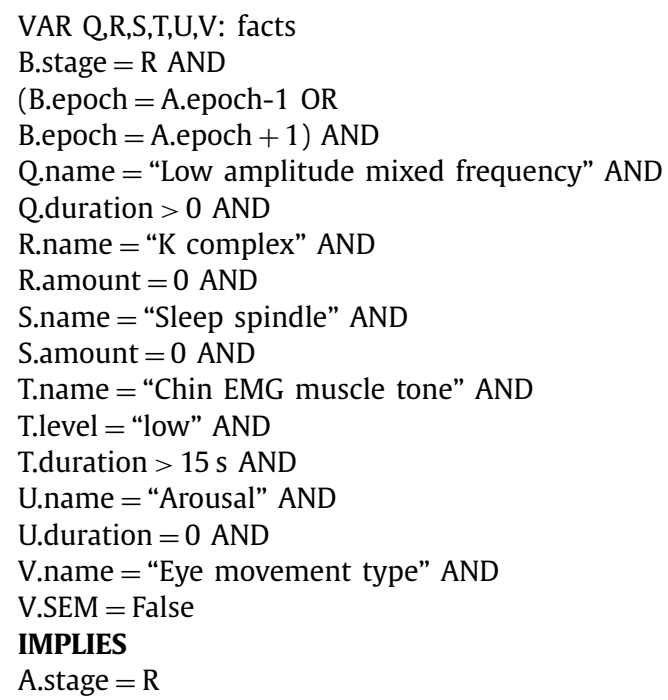

- End-stage scoring rule: A rule that determines the end of a definitive stage.

Example for an adult patient: If an arousal in the previous epoch $_{i}$ interrupts stage $\mathrm{N} 2$ sleep, score subsequent segments of the recording as stage $\mathrm{N} 1$ if there is low amplitude mixed frequency EEG activity without $\mathrm{K}$ complexes or sleep spindles, until there is evidence for another sleep stage.

- Transition-stage scoring rule: A rule that determines the end of a definitive stage and the start of scoring for a different stage.
Example for an adult patient: A transition to stage N1 exists if the previous epoch is scored as stage $\mathrm{R}$ and an arousal occurs followed by low amplitude mixed frequency EEG and SEM.

\subsubsection{Inferential knowledge}

In this section we describe how the static structures are used to implement reasoning processes. The main elements that specify knowledge about inferences are: (a) inferences, (b) knowledge roles, and (c) transfer functions. Inferences, which are the link between the tasks/methods and the domain schema, are perfectly defined on the basis of inputs, outputs and static knowledge, so there is no need to specify internal control. For each role used in an inference, a mapping with an object of the domain exists (Fig. 11).

For knowledge about inferences the lowest level of functional decomposition is described, with each inference fully described by means of a declarative specification of inputs and outputs (dynamic roles). The internal process is considered to be a black box and so is not of interest in modeling the knowledge.

The methods for the hypnogram construction sub-task (identify and instantiate sleep stages) are decomposed into the five functions (inferences) described in Section 6.1 and on the basis of these inferences the design is modified.

\section{Knowledge model: knowledge refinement stage}

This activity has two distinct phases: (a) knowledge bases are expanded, and (b) validation is performed by means of a walkthrough.

\subsection{Knowledge model validation}

Below the process for temporal correlation of the symbolic information is described. For each step, the inferences involved are indicated and the process is briefly illustrated using examples.

STEP 1: The PSG recording is introduced into the system. For example, instances of alpha rhythm (posterior dominant rhythm), eye blinks, reading eye movements and rapid eye movements (REM) events that occur during epoch 1 are introduced into the KBS.

STEP 2: The conditions that must be satisfied for an epoch (based on events that occur inside it) to be considered as belonging to a particular sleep stage are specified (specify). For example, we consider these two conditions: 


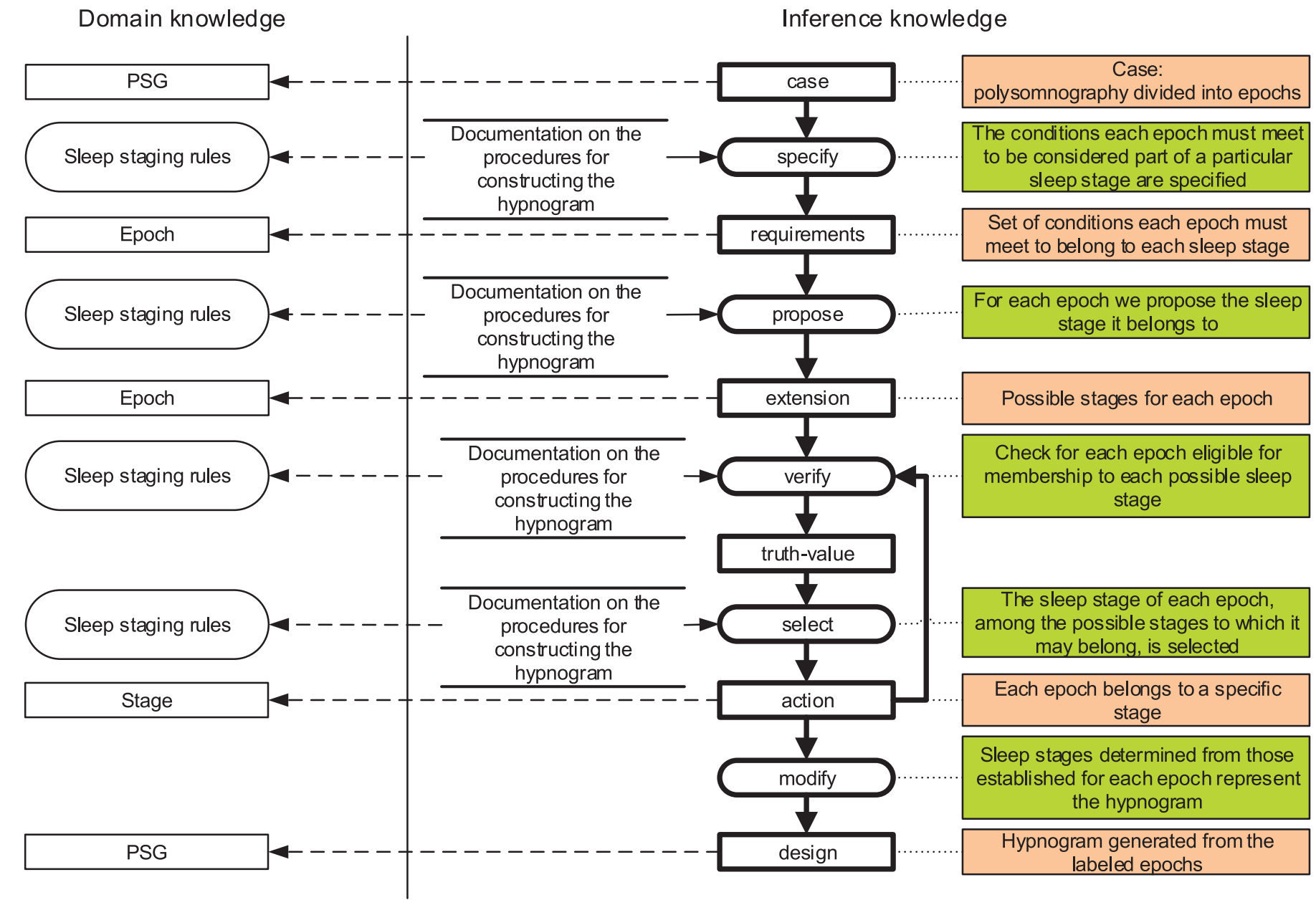

Fig. 11. Mapping of the inference diagram for the hypnogram construction task.

- Condition 1: Score epochs as stage $\mathrm{W}$ when more than $50 \%$ of the epoch contains: eye blinks $(00.5-2 \mathrm{~Hz})$, rapid eye movements associated with normal or high chin muscle tone, or reading eye movements

- Condition 2: In individuals who generate alpha rhythm, score stage $\mathrm{Nl}$ if the alpha rhythm is attenuated and replaced by low amplitude, mixed-frequency activity for more than $50 \%$ of the epoch.

STEP 3: Proposed to each epoch are the sleep stages it may belong to (propose). For example, analysis of the events that occur during epoch 1 would indicate that these may belong to phase $\mathrm{W}$ or phase N1.

STEP 4: Verified for each epoch is that membership conditions are satisfied at every possible sleep stage it may belong to (verify). For example, the conditions of membership of epoch 1 to phases $\mathrm{W}$ and $\mathrm{N} 1$ are verified, as specified above.

STEP 5: An epoch is assigned to a stage taking into account possible stages it may belong to (select). For example, if it is determined that epoch 1 corresponds to phase W, then STEP 4 and STEP 5 are repeated until all the possible sleep stages are considered and only then is STEP 6 launched.

STEP 6: The sleep stages, defined from those established for each epoch, are represented in the hypnogram (modify). With the establishment of the sleep stages to which each epoch belongs, adjacent epochs belonging to the same stage are grouped together and the corresponding sleep stages are instantiated.

\subsection{Knowledge base completion}

Knowledge base implementation was completed through a knowledge acquisition process in accordance with the proposed model, with detailed information about the rules needed for reasoning and system development.

\section{Communication model}

This model, which like the knowledge model, is independent of the implementation, describes the information object transactions to be performed by the agents who collaborate in performing the tasks. Its aim is the detailed specification of processes that transfer information/knowledge between the different agents of KBS, i.e., this model details which information is transmitted and between which agents. It also acts as a higher-level control over the execution of a task.

The communication model is divided into three components. The first component is the communications plan, which describes the entire dialogue between two agents. The second component is the transaction - the basic atomic element of the communication model. Transactions take place between two task sheets implemented by different agents. The third component is the information exchange specification that describes (should this be necessary) the internal structure of a message.

From the decomposition of system processes it can be observed that leaf tasks are as follows: (1) perform PSG, (2) analyze pul- 
Sleep unit

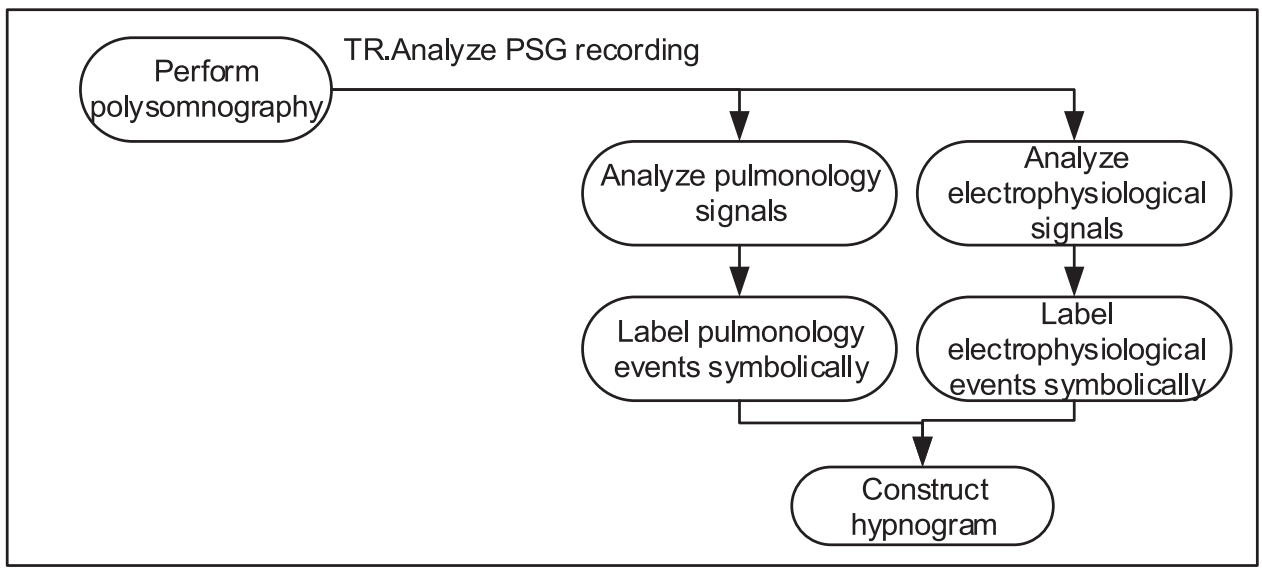

Fig. 12. Dialogue diagram for the communications plan.

monology signals, (3) analyze electrophysiological signals, (4) label pulmonology events symbolically, (5) label electrophysiological events symbolically, and (6) construct the hypnogram. Described for each of these tasks are objects in input and output and the agents involved and information exchanges between agents for each of the tasks and/or transfer functions are analyzed. Fig. 12 shows a dialog diagram depicting these steps.

Once the design knowledge model has been completed, the next step (according to CommonKADS methodology) is the construction of the design model. The design model will be constructed according to the method of signal analysis and detection and classification of signal patterns to be considered, so that the final system enables a better update to changes in rules of hypnogram construction or methods of signal analysis.

\section{Discussion}

The hypnogram is mainly used as a qualitative method for indicating the duration of each sleep stage and the number of transitions between stages. When analyzed in conjunction with records of certain physiological processes important quantitative measures of sleep quality are obtained.

Two basic processes underpin the construction of a hypnogram: (1) events indicated by PSG signals are correctly identified and classified, and (2) on the basis of the detected events, the patient's different sleep stages are identified.

With regard to signal identification and classification, it is necessary that the recording of signals and the corresponding interpretation mechanisms be as reliable possible and in accordance with the AASM guidelines. So, much work has recently focused on correct identification of relevant events (such as arousals) and signal patterns (especially of sleep spindles, $\mathrm{K}$ complexes, vertex sharp waves and sawtooth waves), resulting in improvements to automatic sleep analysis and hyponogram construction methods. Developing KBSs using the proposed approach eases the comparison between methods and the analysis of the effectiveness of the proposed improvements thanks to conceptual modeling. Conceptual modeling via the knowledge and communication models means that high-level KBS design can be isolated from KBS implementation. The methodology also provides a project management and planning framework to define a spiral lifecycle of development.

Regarding the existing implementations for automatic analysis of sleep and its stages, the developed knowledge model is easily applied to those methods that use signal characteristic extrac- tion by frequency analysis and frequency over time. This is because the separation between signal processing and the treatment of the events obtained from that processing is part of the requirements of the proposed model. However, AASM rules cannot be directly applied in some methods that use parametric and non-parametric classification of sleep events and stages, such as artificial neural networks. In these cases, it would be necessary to feed the neural network with cases diagnosed by experts strictly applying the AASM rules.

Another contribution of our work comes from the idea of knowledge reuse. CommonKADS defines templates that constitutes predefined reusable knowledge models that have proven to work in the past. In our case, we have chosen the "configuration template" in order to build the inference diagram for the hypnogram construction task. However, CommonKADS templates tend to be very general and usually you have to adapt them to the characteristics of your specific task. For example, we have adapted only a part of the configuration template to our task of building a hypnogram based on several signals that we divide in epochs and classify each epoch into a category (in this case sleep stages).

Our solution was detailed in this paper by an inference diagram that includes knowledge dynamic roles and inferences that were annotated to explain its correspondence in our specific domain. We believe that this solution can be applied to other similar problems building what is called "patterns of knowledge-intensive tasks" that will help designers reuse successful designs by basing new designs on prior experience.

Thanks to the work presented, we have obtained a knowledge model for the development of a framework for hypnogram construction that has the advantage of isolating the process of signal processing from the sleep stages identification. Thus, the validation of the systems developed using our KBS can be homogenized, with the result that hypnograms and their results become comparable, independently of the PSG signal analysis techniques. In this context, the CommonKADS methodology is a powerful tool for knowledge acquisition and KBS development.

A possible disadvantage of the proposed knowledge model is the increased complexity of the systems that are built using it as a base. However, this is compensated by the advantages that are obtained from its application.

On the other hand, implementing rules published by the AASM involves treatment of signals using a division of the records at intervals, continuing the methodology established by the R\&K rules. This division limits the ability to record and analyze events lasting less than $30 \mathrm{~s}$, and ignores the fact that the evolution of the bio- 
logical processes occurring continuously, which implies a smooth transition between sleep stages [1]. To overcome the limitations of a system based on time intervals, several methods have been developed recently. For example, Alvarez-Estevez et al. developed a method for the automatic analysis of the macrostructure of sleep continuously [33]. This method is based on the use of fuzzy inference to avoid categorical classifications, and permits the representation of smooth transitions of sleep through their different stages. The results of this method suggest the desirability of advancing in the study of mechanisms dealing with sleep signals as a continuous record.

\section{Conclusions}

This approach has two major features compared to other methods: (1) different KBS results can be compared because PSG signal processing and signal processing techniques are independent, and (2) the hypnogram construction process is structured in such a way that it can be easily adapted to future changes in sleep stage identification and classification guidelines independently of the development environment.

The inclusion of this intelligent sleep classifier module in an integrated system results in a perfectly autonomous product, potentially ready for commercialization.

Future directions of the research include: (1) the application of a proposed KBS to build a hypnogram using different methods to analyze PSG signals, and (2) the comparison of results as well as the application of the proposed method in clinical surroundings and the detection of sleep disorders or the diagnosis of the syndrome of sleep apnoea.

\section{Conflict of interest}

The authors declare that they have no conflict of interest.

\section{Acknowledgments}

This work has been supported by Xunta de Galicia, Spain (excellence groups program GRC2014/035) and by the Ministerio de Economía y Competitividad, Spain (research project TIN201340686-P, MINECO, co-funded by FEDER).

\section{References}

[1] A. Fernández-Leal, V. Moret-Bonillo, M.J. Cabrero-Canosa, et al., Towards the standardization of hypnogram construction for sleep analysis, in: E. Kyriacou, et al. (Eds.), XIV Mediterranean Conference on Medical and Biological Engineering and Computing 2016, IFMBE Proceedings, 57, Springer International Publishing, Switzerland, 2016, pp. 859-863, doi:10.1007/978-3-319-32703-7_ 168.

[2] G. Schreiber, H. Akkermans, A. Anjewierden, R. de Hoog, N. Shadbolt, W. Van de Velde, et al., Knowledge engineering and management, The CommonKADS Methodology, 2000 ISBN 0-262-19300-0.

[3] A. Alonso Betanzos, B. Guijarro Berdiñas, A. Lozano Tello, J.T. Palma Méndez, M.J. Taboada Iglesias, Ingeniería del Conocimiento, Aspectos Metodológicos, Pearson Educación S.A, 2004 84-205-4192-3.

[4] RB Berry, R Brooks, CE Gamaldo, SM Harding, RM Lloyd, CL Marcus, BV Vaughn, The AASM Manual for the Scoring of Sleep and Associated Events: Rules, Terminology and Technical Specifications, American Academy of Sleep Medicine, Darien, Illinois, 2015 Version 2.2. www.aasmnet.org.

[5] ThomasF. Collura, History and evolution of electroencephalographic instruments and techniques, J. Clin. Neurophysiol 10 (4) (1993) 476-504.

[6] W.Grey Walter, The location of cerebral tumors by electro-encephalography, Lancet 228 (5893) (1936) 305-308 8 August 1936.

[7] W. Walter, V. Dovey, Electro-encephalography in cases of sub-cortical tumour, J. Neurol. Neurosurg. Psychiatry 7 (1944) 57-65.

[8] A.L. Loomis, E.N. Harvey, G.A. Hobart, Cerebral states during sleep, as studied by human brain potentials, J. Exp. Psychol. 21 (2) (1937) 127-144, doi:10.1037/ h0057431.

[9] A.L. Loomis, E.N. Harvey, G.A. Hobart, Electrical potentials of the human brain, J. Exp. Psychol. 19 (1936) 249-279.
[10] A.L. Loomis, E.N. Harvey, G.A. Hobart, Distribution of disturbance patterns in the human electroencephalogram, with special reference to sleep, J. Neurophysiol 1 (1938) 413-430.

[11] WT. Liberson, Problem of sleep and mental disease, Digest Neurol. Psychiatry 12 (1944) 93-108

[12] E Aserinsky, N Kleitman, Regularly occurring periods of eye motility, and concomitant phenomena, during sleep, Science, New Series 118 (3062) (1953) 273-274.

[13] W Dement, N Kleitman, Cyclic variations in EEG during sleep and their relation to eye movements, body motility, and dreaming, ElectroencephClin. Neurophysiol. 9 (4) (1957) 673-690 PMID 13480240, doi:10.1016/0013-4694(57) 90088-3.

[14] R.J. Berger, P. Olley, I. Oswald, The eec, eye-movements and dreams of the blind, Q. J. Exp. Psychol. 14 (3) (1962) 183-186, doi:10.1080 17470216208416534

[15] B. Schwartz, EEG et mouvement oculaires dans le sommeil de muit, ElectroencephClin. Neurophysiol. 14 (1) (February 1962) 126-128.

[16] M.A.B. Brazier, et al., Proposal for an LEG terminology by the terminology committee of the international federation for electroencephalography and clinical neurophysiology, Electroenceph. Clin. Neurophysiol. 13 (1961) 646-650.

[17] A. Reschtschaffen, A. Kales, A Manual of Standarized Terminology, Techniques, and Scoring System for Sleep Stages of Human Subjects, Washington Public Health Service. US Government Printing Office, Washington DC, 1968.

[18] Sleep Computing Committee of the Japanese Society of Sleep Research Society (JSSR). Tadao Hori, Proposed supplements and amendments to 'A Manual of Standardized Terminology, Techniques and Scoring System for Sleep Stages of Human Subjects', the Rechtschaffen \& Kales (1968) standard, Psychiatry Clin. Neurosci. 55 (2001) 305-310.

[19] Task Force 'Scoring of Polysomnographic Recordings' of the German Sleep Society (DGSM): Hartmut Schulz et al. (2006). A review of sleep EEG patterns Part I: A Compilation of Amended Rules for Their Visual Recognition according to Rechtschaffen and Kales.

[20] C Iber, S Ancoli-Israel, AL Chesson, SF Quan, The AASM Manual for the Scoring of Sleep and Associated Events: Rules, Terminology, and Technical Specification, American Academy of Sleep Medicine, Westchester, IL, 2007 Version 10-9657220-4-X

[21] R Berry, R Brooks, C Gamaldo, et al., The AASM Manual for the Scoring of Sleep and Associated Events: Rules, Terminology, and Technical Specification, American Academy of Sleep, Darien, IL, 2012 Version 2.0

[22] R Berry, R Brooks, C Gamaldo, et al., The AASM Manual for the Scoring of Sleep and Associated Events: Rules, Terminology, and Technical Specification, American Academy of Sleep, Darien, IL, 2014 Version 2.1.

[23] S Khalighi, T Sousa, G Pires, U Nunes, Automatic sleep staging: A computer assisted approach for optimal combination of features and polysomnographic channels, Expert Syst. Appl. 40 (2013) 7046-7059.

[24] L. Zoubek, S. Charbonnier, S. Lesecq, A. Buguet, F. Chapotot, Feature selection for sleep/wake stages classification using data driven methods, Biomed. Signal Process. Control 2 (Jul 2007) 171-179.

[25] H.G. Jo, J.Y. Park, C.K. Lee, S.K. An, S.K. Yoo, Genetic fuzzy classifier for sleep stage identification, Comput. Biol. Med. 40 (Jul 2010) 629-634.

[26] W.C. Tang, S.W. Lu, C.M. Tsai, C.Y. Kao, H.H. Lee, Harmonic parameters with HHT and wavelet transform for automatic sleep stages scoring, Proc. World Acad. Sci. Eng. Technol. 22 (2007) 414-417.

[27] L. Fraiwan, K. Lweesy, N. Khasawneh, H. Wenz, H. Dickhaus, Automated sleep stage identification system based on time-frequency analysis of a single EEC channel and random forest classifier, Comput. Methods Programs Biomed. 108 (1) (2012) 10-19

[28] S. Gunes, K. Polat, S. Yosunkaya, Efficient sleep stage recognition system based on EEG signal using k-means clustering based feature weighting, Expert Syst. Appl. 37 (Dec 2010) 7922-7928.

[29] S. Khalighi, T. Sousa, D. Oliveira, G. Pires, U. Nunes, Efficient feature selection for sleep staging based on maximal overlap discrete wavelet transform and SVM, in: Annual International Conference of the IEEE Engineering in Medicine and Biology Society (EMBC), 2011, pp. 3306-3309.

[30] S. Khalighi, T. Sousa, U. Nunes, Adaptive automatic sleep stage classification under covariate shift, in: 2012 Annual International Conference of the IEEE Engineering in Medicine and Biology Society (EMBC), 2012, pp. 2259-2262.

[31] M. Ronzhina, O. Janousek, J. Kolarova, M. Novakova, P. Honzik, I. Provaznik Sleep scoring using artificial neural networks, Sleep Med. Rev. 16 (Jun 2012) 251-263.

[32] L. Fraiwan, K. Lweesy, N. Khasawneh, M. Fraiwan, H. Wenz, H. Dickhaus, Classification of sleep stages using multi-wavelet time frequency entropy and LDA Methods Inf. Med. 49 (2010) 230-237.

[33] D. Álvarez-Estévez, J.M. Fernández-Pastoriza, E. Hernández-Pereira, V. Moret-Bonillo, A method for the automatic analysis of the sleep macrostructure in continuum, Expert Syst. Appl. 40 (2013) 1796-1803 2013.

[34] V.C.F. Helland, A. Gapelyuk, A. Suhrbier, M. Riedl, T. Penzel, J. Kurths, N. Wessel, Investigation of an automatic sleep stage classification by means of multiscorer hypnogram, Methods Inf. Med. 49 (2010) 467-472.

[35] M.E. Tagluk, N. Sezgin, M. Akin, Estimation of sleep stages by an artificial neural network employing EEG, EMG and EOG, J. Med. Syst. 34 (Aug 2010) $717-725$.

[36] F. Chapotot, G. Becq, Automated sleep-wake staging combining robust feature extraction, artificial neural network classification, and flexible decision rules, Int. J. Adapt. Control Signal Process. 24 (May) (2010) 409-423. 
[37] A Nonclercq, C Urbain, D Verheulpen, C Decaestecker, P Van Bogaert, $\mathrm{P}$ Peigneux, Sleep spindle detection through amplitude-frequency normal modelling, J. Neurosci. Methods. 214 (2) (2013) 192-203, doi:10.1016/j. jneumeth.2013.01.015.

[38] T.A. Camilleri, K.P. Camilleri, S.G. Fabri, Automatic detection of spindles and Kcomplexes in sleep EEG using switching multiple models, Biomed. Signal Process. Control 10 (2014) 117-127, doi:10.1016/j.bspc.2014.01.010.
[39] E Hernández-Pereira, V Bolón-Canedo, N Sánchez-Maroño, D Álvarez-Estévez, V Moret-Bonillo, A Alonso-Betanzos, A comparison of performance of K-complex classification methods using feature selection, Inf. Sci. 328 (2016) 1-14.

[40] D. Alvarez-Estevez, V. Moret-Bonillo, Identification of electroencephalographic arousals in multichannel sleep recordings, IEEE Trans. Biomed. Eng. 58 (1) (2011) 54-63. http://doi.org/10.1109/TBME.2010.2075930. 